\title{
Customized Ocular Prosthesis
}

\section{Anuradha Ganpat Mohite}

\begin{abstract}
Artificial replacement of the lost eye is done with an ocular prosthesis. Loss of eye or any other facial structures affect the physical, emotional and psychological well being of a person. Aim of the maxillofacial prosthesis that will replace such structures will improve patients' esthetics, restore and maintain health of these structures and, thereby provide physical and mental well being. Accurate impression to duplicate the contours of the defect site is required for the fabrication of custom made prosthesis. A case report of customized ocular prosthesis is described here.
\end{abstract}

Keywords: Ocular defect, Customized ocular prosthesis, Split cast mold, Scleral wax pattern, Iris, Scleral blank.

How to cite this article: Mohite AG. Customized Ocular Prosthesis. J Contemp Dent 2015;5(1):53-59.

\section{Source of support: Nil}

Conflict of interest: None

\section{INTRODUCTION}

Eye is a vital organ not only in terms of vision but also an important component of facial expression. ${ }^{1}$ Loss of eye or any other facial structures affect the physical, emotional and psychological well being of a person. In such a situation, it becomes important for immediate replacement of the lost structures to improve patient's mental well being and improve social acceptance. ${ }^{1}$ Artificial replacement of the eye is done with the help of an ocular prosthesis which can be customized according to the defect for that particular patient. Surgical procedures adopted for removal of eye are classified by Peyman, Saunders and Goldberg into three general categories: Enucleation, Evisceration and extenteration. ${ }^{2}$ Impression technique for eye prosthesis varies according to the type of the defect. ${ }^{1}$ An accurate impression will reproduce the defect exactly, thereby producing an accurately fitting and retentive prosthesis. ${ }^{3}$ Customized ocular prosthesis have more esthetic and precise results compared to stock eye prosthesis. ${ }^{1}$ Treatment options for an ocular defect may

\section{Lecturer}

Department of Prosthodontics, MGM Dental College and Hospital, Navi Mumbai, Maharashtra, India

Corresponding Author: Anuradha Ganpat Mohite, Lecturer Department of Prosthodontics, MGM Dental College and Hospital Navi Mumbai, Maharashtra, India, Phone: 02227426604, e-mail: dr.anu07@gmail.com involve implants or acrylic resin prosthesis. Fabricating implant prosthesis may require more of technical skill. Since, bone is very thin in this region, it is difficult to place implants. Also, patient being very petit implants are not advisable. Moreover, implants may not be advisable in patients with poor socioeconomic status. A customized acrylic resin ocular prosthesis is a viable alternative for such patients. ${ }^{3}$

\section{CASE REPORT}

A 16-year-old female patient reported with a ocular defect of right eye and needed replacement for the same for esthetic concern (Figs 1A and B). Patient gave history of whitish discoloration in her right eye 6 years back which gradually increased. It was associated with mild pain and redness which eventually progressed. The lesion was diagnosed as Atrophic Bulbi and was enucleated, globe was removed and attached portion of optic nerve was excised leaving behind the upper and lower eyelids intact. After the healing period of 3 months, the patient was referred by the ophthalmic surgeon to the Department of prosthodontics for fabrication of ocular prosthesis. On examination of the socket, well healed tissue bed and absence of infection was observed (Figs 1A and B).

\section{PROCEDURE}

\section{Impression of the Eye Socket}

Patient was instructed to gaze directly forward at a fixed point atleast 6 feet away. This will provide impression of the site with the muscles captured in neutral position. Alginate was mixed and loaded in a disposable plastic syringe and expressed into the eye socket under the eyelids $^{4}$ (Fig. 2A). Gauze was placed over the alginate while it was setting for anchorage of the dental plaster (Fig. 2B). Dental plaster was mixed in thick consistency and placed over the gauze as backing for support of the impression (Fig. 2C). ${ }^{5}$ The impression was removed from the socket after both alginate and plaster was set taking care to remove the impression from the lower, shallower eyelid sulcus first, then rotated out from the deeper upper eyelid sulcus to prevent distortion of the impression ${ }^{4}$ (Fig. 3).

\section{Boxing of the Impression}

The impression was boxed with boxing wax and poured in dental stone to obtain the first cast. ${ }^{4}$ This was done 
to preserve the borders for precise fabrication of the prosthesis.

\section{Final Impression}

On the first cast, a special tray with self-cure acrylic resin was fabricated with a hollow tube attached at the center of the special tray for injecting the impression material at a later stage. Tray adhesive was applied to the special tray on both the sides (Fig. 4). Final impression was taken using Light body impression material ${ }^{1}$ (Figs 5A and B). Light body was mixed on a mixing pad and loaded in the syringe to be injected through the tube attached to the special tray into the eye socket. Material was allowed to set. The impression was removed from the eye socket

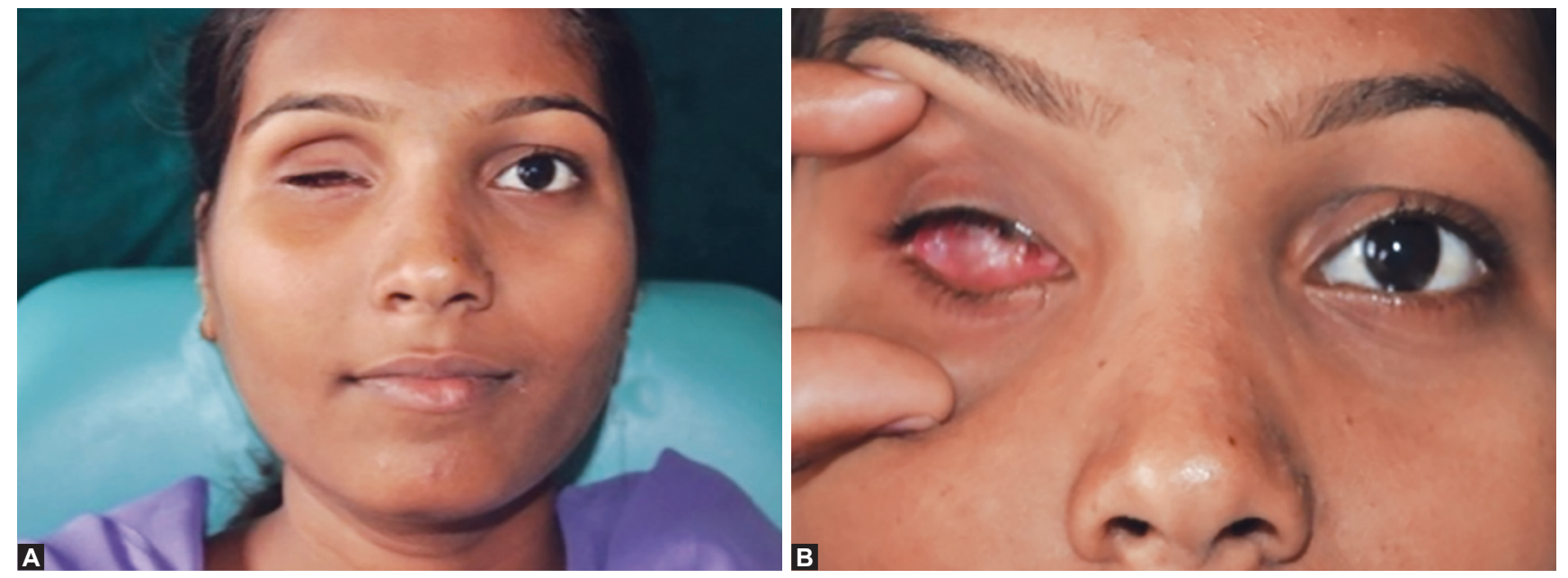

Figs $1 \mathrm{~A}$ and $\mathrm{B}$ : Preoperative view of the patient

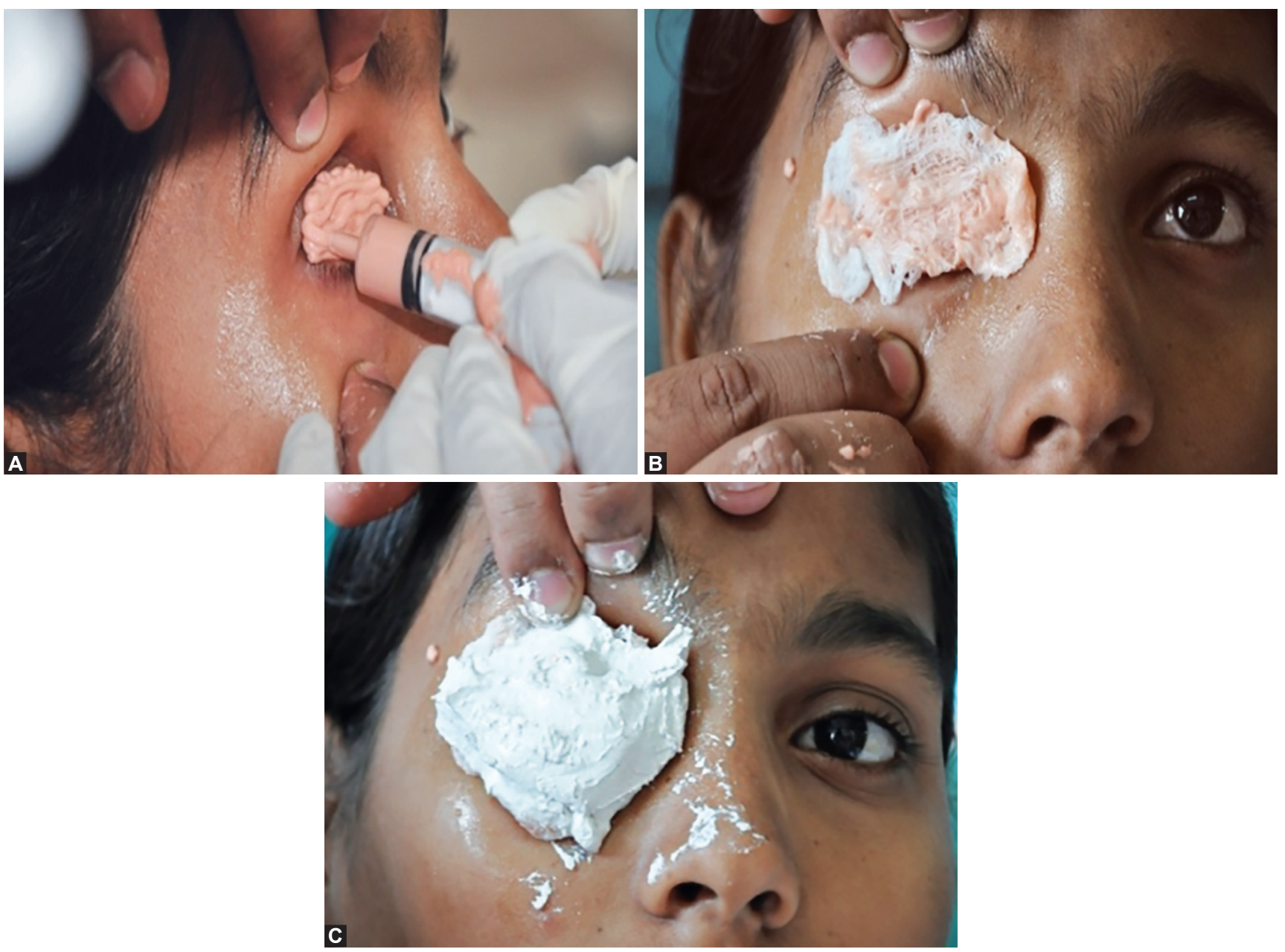

Figs 2A to C: (A) Alginate expressed into eye socket, (B) gauze placed over alginate, (C) dental plaster backing over alginate impression 
and pored in Die stone to obtain a split cast mold ${ }^{1}$ (Fig. 6). The impression was pored upto the height of contour to obtain the lower half of the split cast mold. After the die stone was set, separating medium was applied and second half or remainder of the impression was pored covering the entire impression. After the die stone was set, the two halves of the cast were separated and the

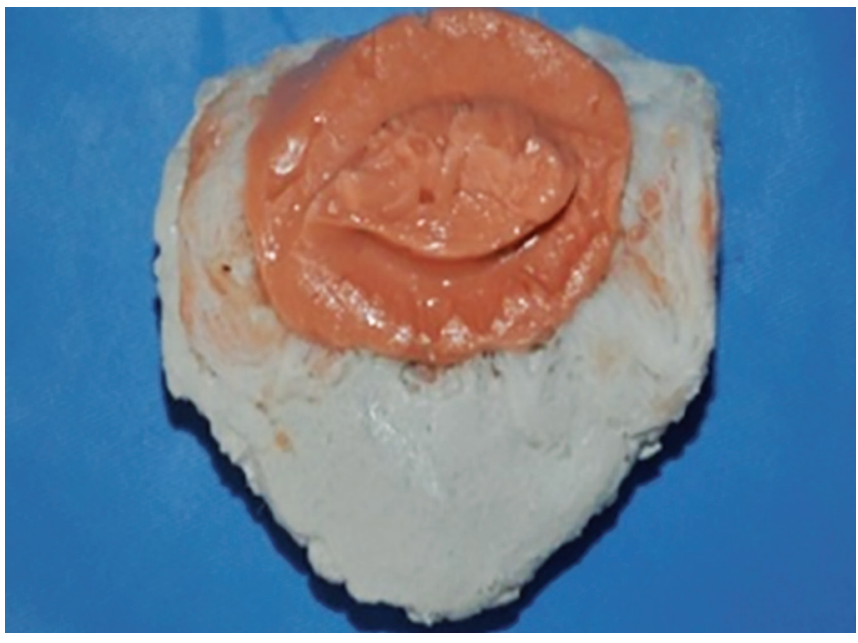

Fig. 3: Impression of eye socket

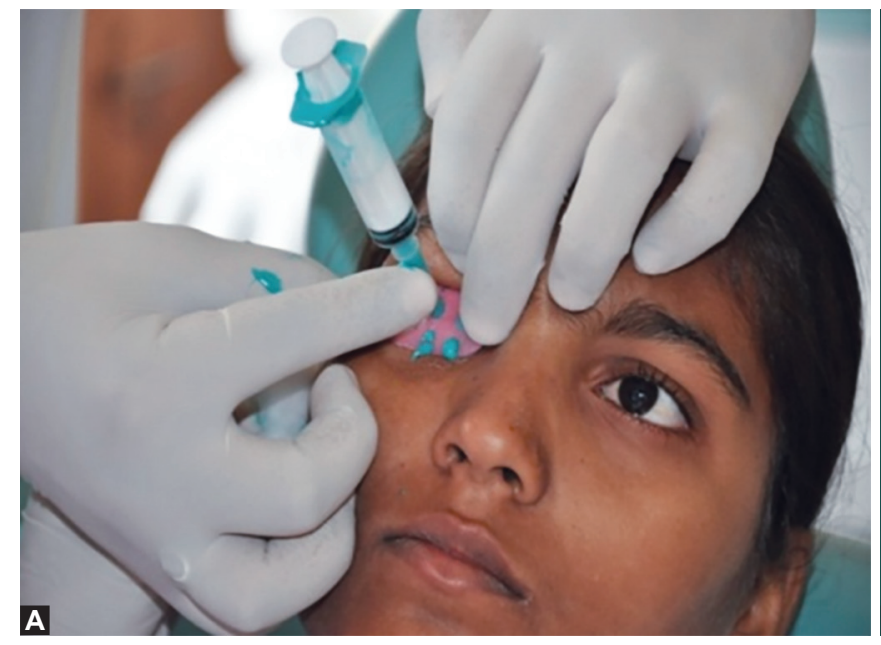

Figs $5 \mathrm{~A}$ and $\mathrm{B}$ : Final impression

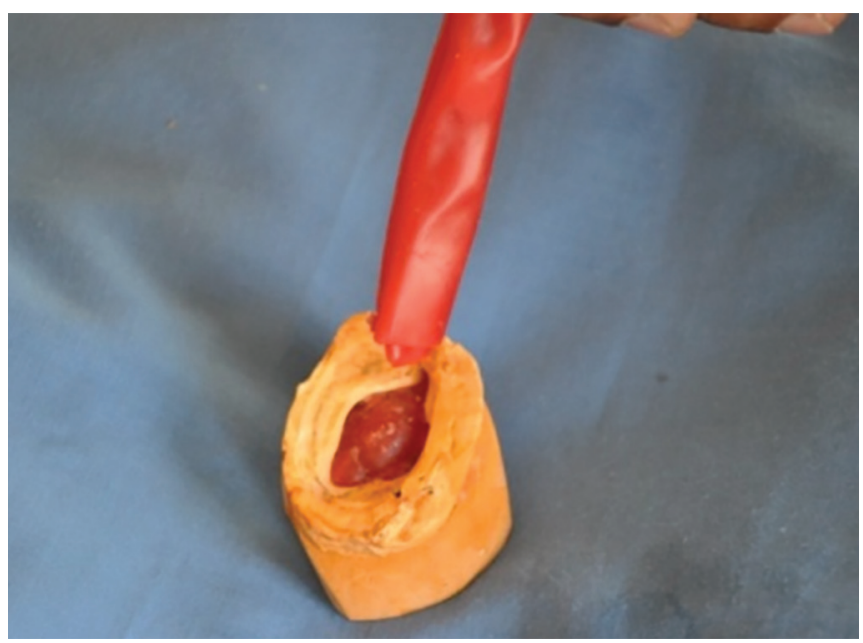

Fig. 6: Split cast mold final impression was retrieved to obtain a mold space for fabrication of scleral wax pattern. ${ }^{4}$

\section{Scleral Wax Pattern Fabrication}

The inner surface of the split cast was coated with separating medium. The cast was reassembled and defect site was filled with molten modelling wax. ${ }^{5}$ The two halves

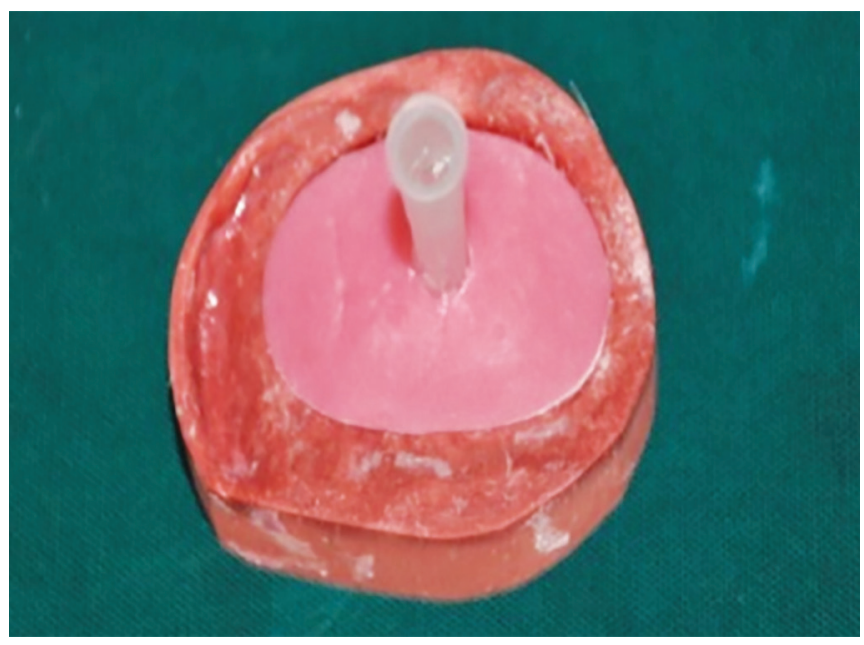

Fig. 4: Special tray with hollow tube
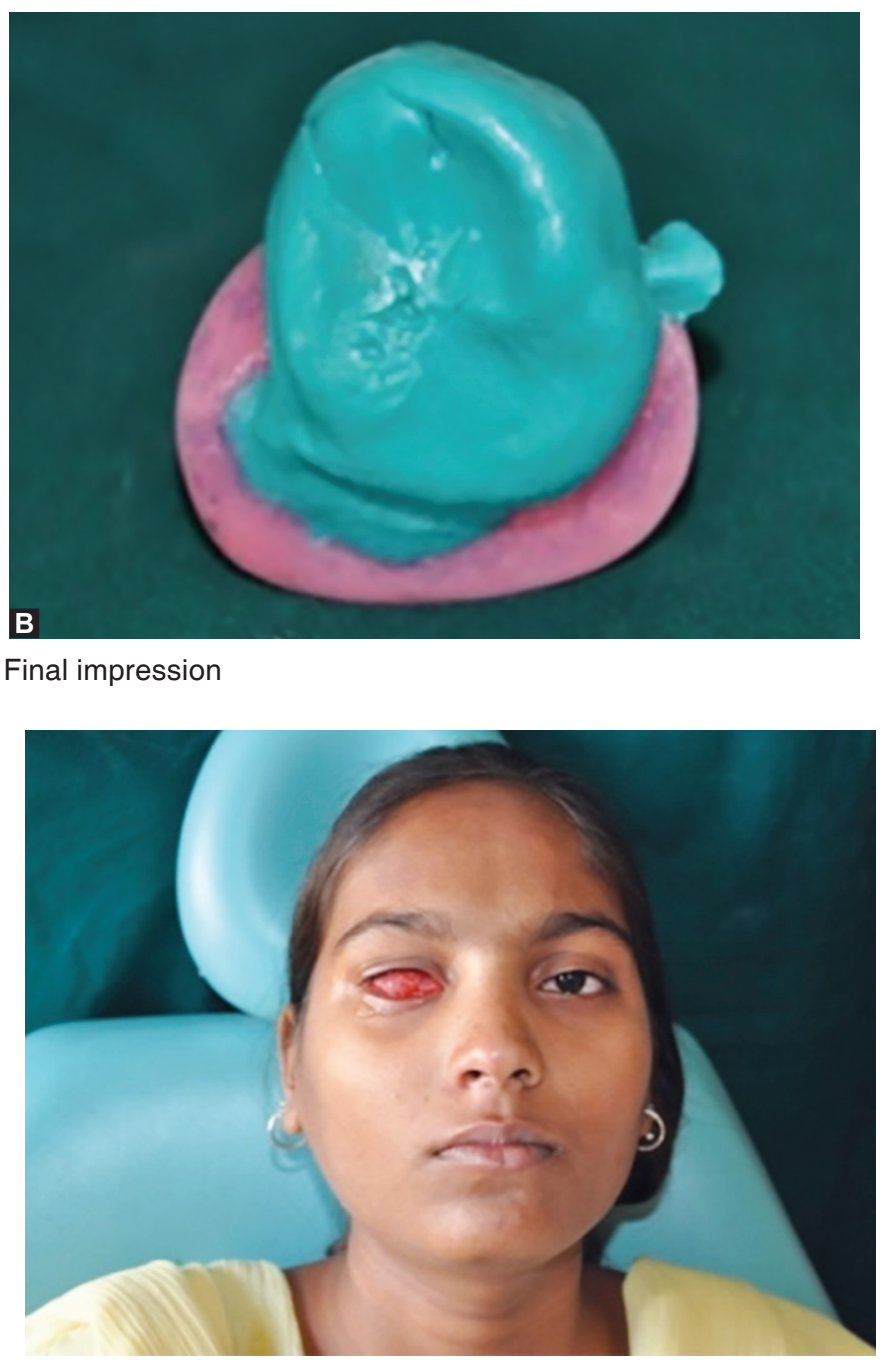

Fig. 7: Trial of scleral wax pattern in eye socket 
of the cast were separated and the wax pattern was retrieved. The wax pattern was highly polished and free from dust and debris before placing it in the eye socket. $^{5}$

\section{Scleral Wax Pattern Try-in}

Scleral wax pattern was tried in the eye socket (Fig. 7). It was evaluated for under-extensions or overextensions. Areas of under-extension were corrected by adding modelling wax. Any over-extensions were reduced. The contour of the scleral wax pattern was evaluated with patient's eyes open and then by manual palpation with the eyes closed. The wax pattern was adjusted until the eyelids completely closed over it. The contours of eyelids with the wax pattern were made to resemble the adjacent natural eye. ${ }^{5}$

\section{Placement of Iris}

Using the natural eye as a guide, the shade and size of the iris was selected. The iris was obtained by trimming a commercially available stock eye (Fig. 8). ${ }^{3}$ The position of the natural iris was determined by asking the patient

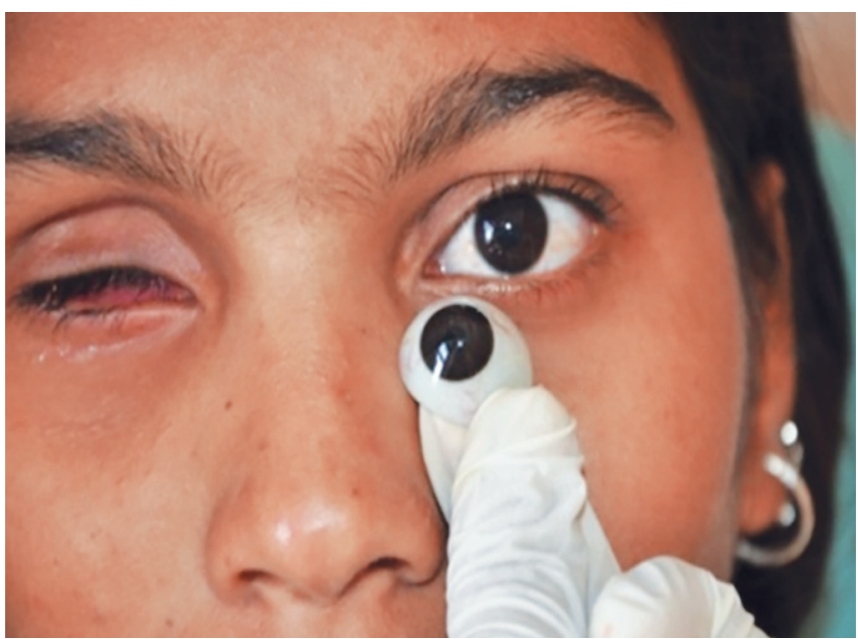

Fig. 8: Distance of iris measured from inner and outer canthus of natural eye

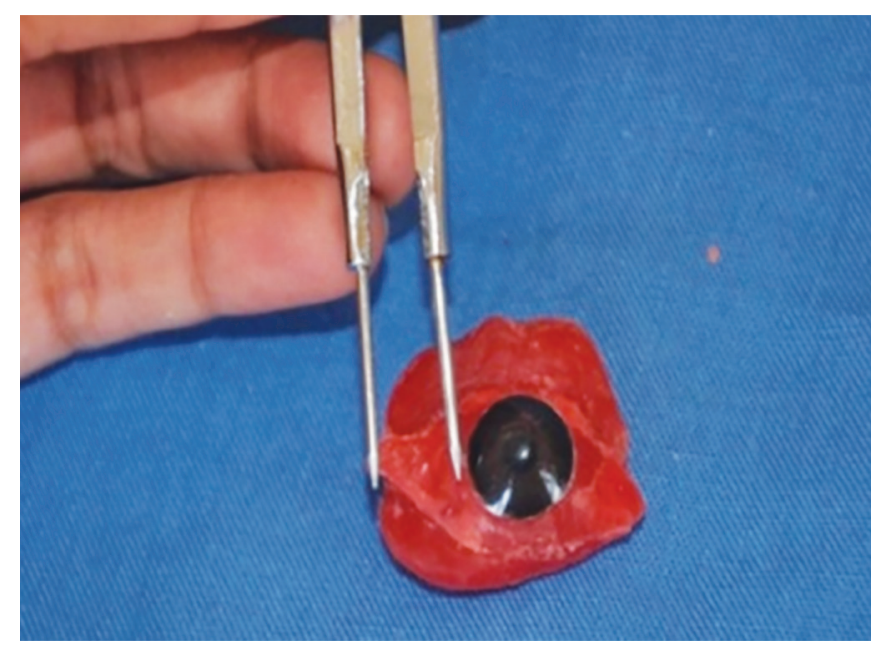

Fig. 10: Stock ocular blank matched with natural eye to look straight ahead to a distant object. ${ }^{5}$ The distance of iris of the natural eye was measured using a divider opposing the iris from the inner canthus to the center of iris and same distance was marked and engraved on the scleral wax pattern. ${ }^{3}$ Then similarly, distance from the outer canthus to the center of the iris was measured using the divider and transferred to the scleral wax pattern ${ }^{5}$ (Fig. 9). The pattern was taken out and the selected iris was placed and adjusted to the horizontal and vertical axis according to the markings engraved on the scleral wax pattern $^{3}$ (Fig. 10). Scleral wax pattern with the iris was tried in the eye socket and checked for symmetry and function by asking the patient to perform various movements (Fig. 11). Patient was asked to move the eyes to the right and left side and look upwards and down. Scleral wax pattern had movements in harmony with adjacent natural eye. ${ }^{5}$

\section{PROCESSING}

Scleral wax pattern was finished to obtain a smooth surface free from dust and debris. The finished scleral wax pattern was invested in the flask (Fig. 12A). The base

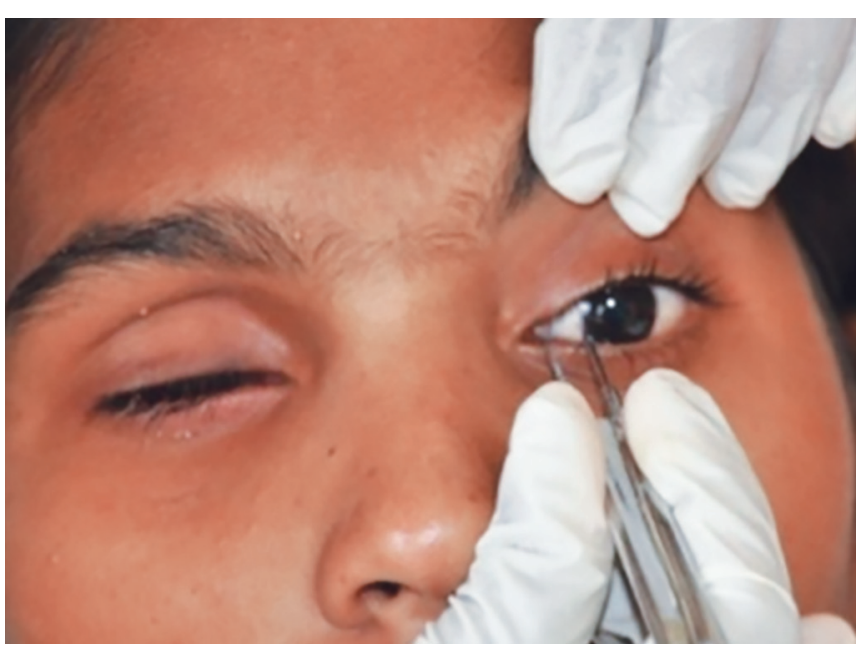

Fig. 9: Placement of iris on scleral wax pattern

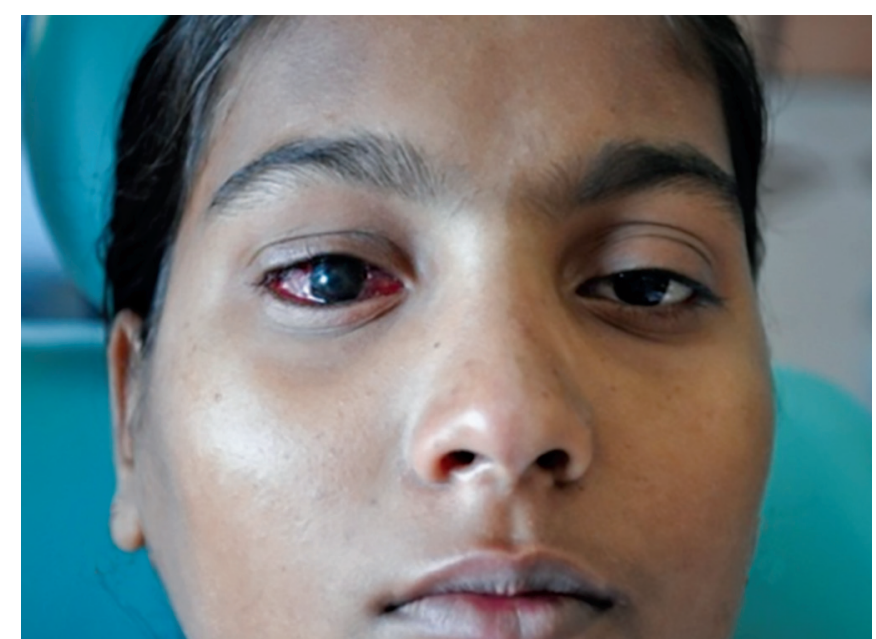

Fig. 11: Trial of scleral wax pattern with iris 
flask was filled with dental plaster and the wax pattern was embedded in the plaster to the height of curvature of the pattern. After, the plaster was set, separating medium was applied and counter-flasking was completed using a mixture of stone and plaster. After it was set, wax was removed by dewaxing (Fig. 12B). The iris and mold were

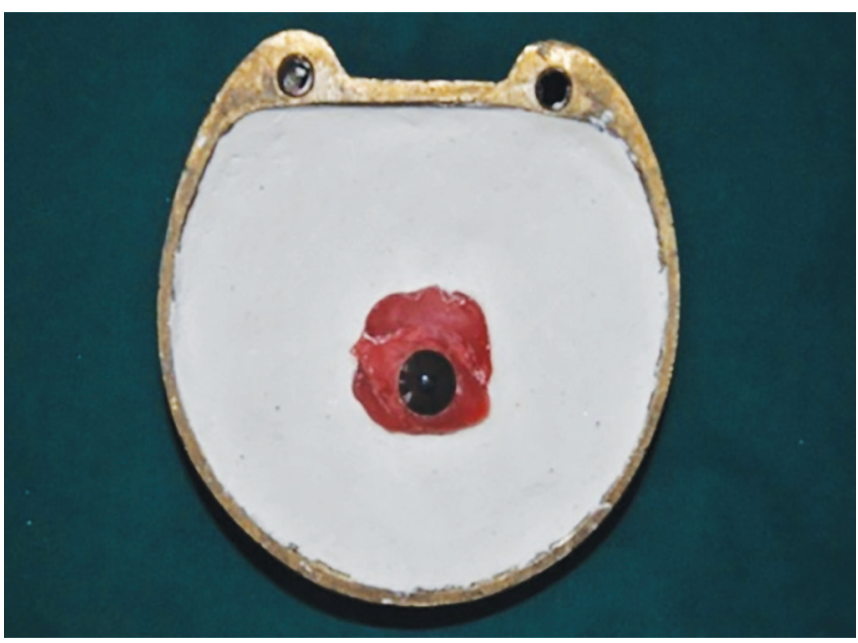

Fig. 12A: Flasking of wax pattern

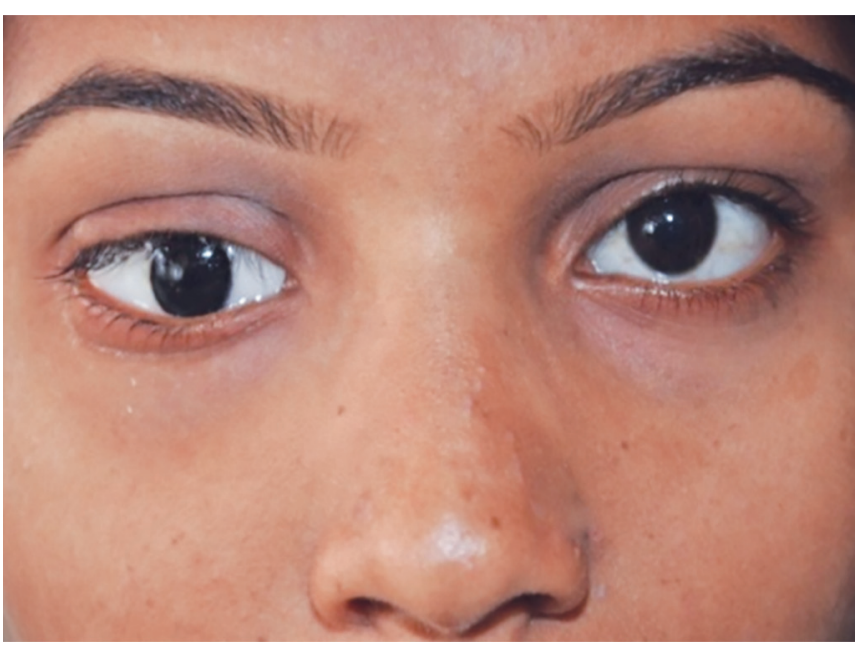

Fig. 13: Trial of scleral blank with iris

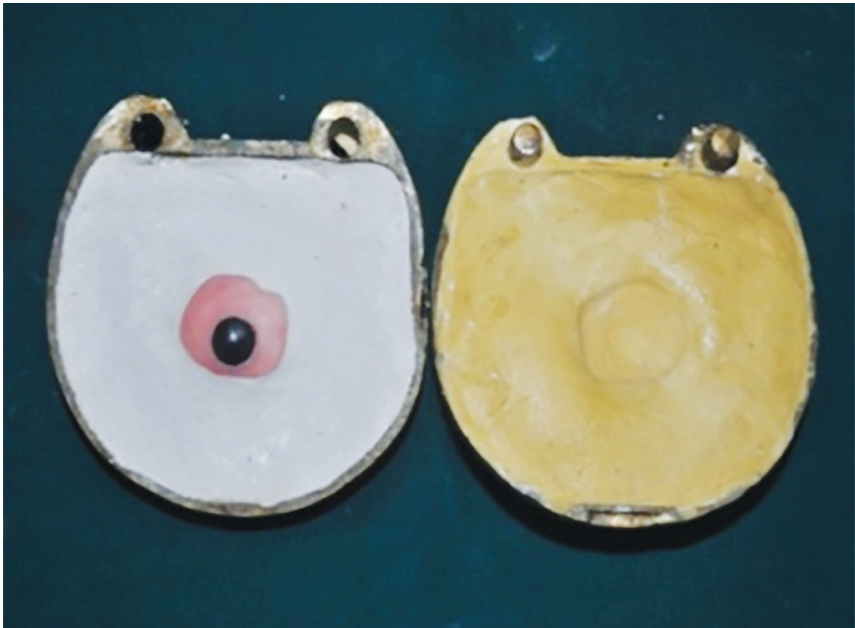

Fig. 15: Spacer wax placed over the corneal region. Prosthesis flasked and dewaxed again cleaned to remove all the wax residue and the mold was painted with separating medium.

\section{PACKING}

Tooth colored acrylic resin polymer and monomer was in the mixed in a ceramic jar in the ratio of 3:1 and packed

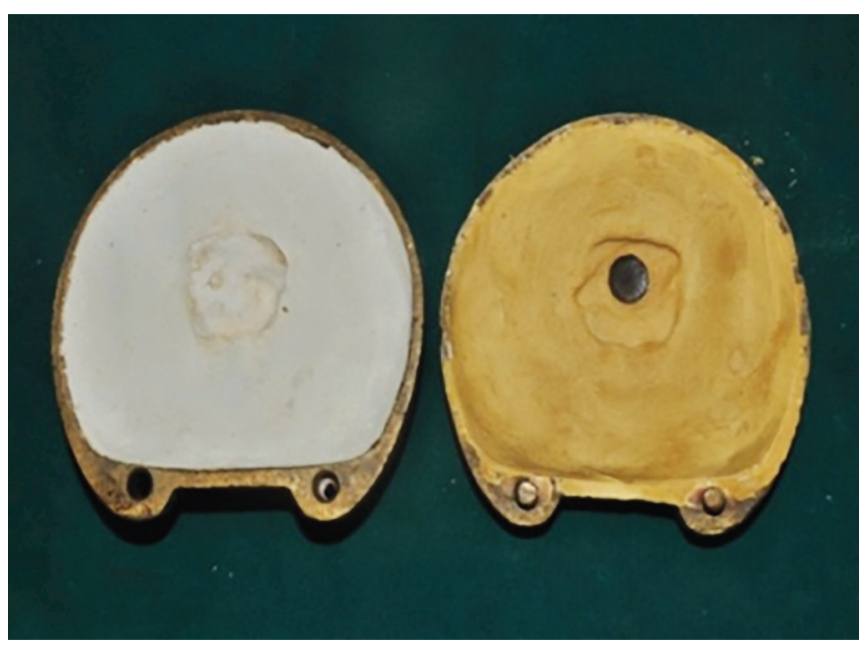

Fig. 12B: Dewaxing of mold

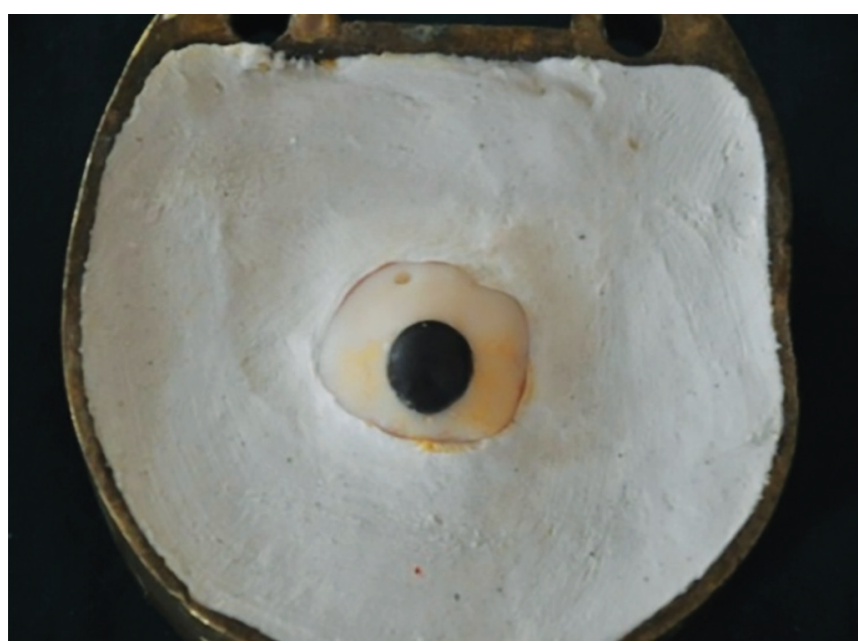

Fig. 14: A combination of yellow and brown acrylic paint applied to the scleral blank

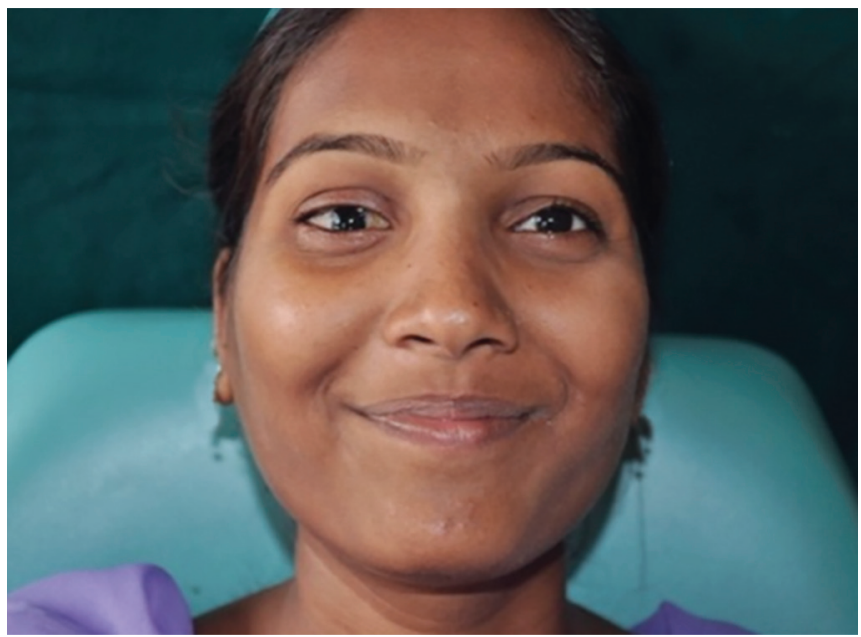

Fig. 16: Placement of final prosthesis in eye socket 
in the mold in the dough stage. The mold was closed and polymerized for 2 hours by short curing cycle. After curing, the mold was allowed to cool down to room temperature. The resulting scleral blank with iris was deflasked carefully. It was trimmed with acrylic trimming burs and polished with Buff and pumice to obtain a smooth surface glossy surface.

\section{TRY-IN OF FINAL PROSTHESIS}

The Scleral blank with iris was tried in the eye socket. (Fig. 13) The position and gaze of the artificial eye was observed. Over-supported areas in the eyelids were modified by removing acrylic resin from the scleral blank by checking for the movements of ocular prosthesis in all directions. It was modified till the prosthesis had movements in harmony with the adjacent natural eye.

\section{CHARACTERIZATION OF THE PROSTHESIS}

After the try-in of the prosthesis, the sclera was characterized to match the natural eye of the patient. A close-up view photograph of the patient was taken to observe the scleral pattern of the patient. ${ }^{5}$ It was seen that there was a slight yellowish hue present on the medial side of the natural eye and few blood vessels. The acrylic resin on the sclera blank was trimmed upto a depth of $1 \mathrm{~mm}$ around the iris with an acrylic trimming bur. ${ }^{5}$ A combination of yellow and brown acrylic paint was mixed with monomer as thinning agent (Fig. 14). This mixture was applied on the trimmed surface to simulate the color of the natural eye. To simulate the blood vessels, red nylon strands were placed on this mixture. Then a layer of spacer wax was placed over the scleral region around the iris keeping the iris exposed. ${ }^{4}$ This was done to cover and protect the pigments and nylon strands from spreading. The prosthesis was again flasked and counter-flasked (Fig. 15). After stone was set, the two halves of the flasks were separated and the layer of spacer wax was carefully removed. To prevent the pigments and nylon strands from spreading, dewaxing was not done. Clear heat cure acrylic was mixed and packed over the scleral blank and polymerized again using short curing cycle. ${ }^{5}$ The ocular prosthesis was carefully retrieved from the flask, trimmed and finely polished to obtain a glass like finish.

\section{PLACEMENT OF FINAL PROSTHESIS}

Before inserting the prosthesis in the socket, it was washed with soap solution and cleaned thoroughly with water before inserting in the eye socket. ${ }^{5}$ A drop of ophthalmic eye solution (Tear Drop; Prescribed by the Opthalmologist) was applied on the surface of the prosthesis to facilitate smooth insertion. The final prosthesis was inserted in the eye socket (Fig. 16). Esthetic appearance of the prosthesis was compared with the natural eye (Fig. 17). Harmonious movement of the prosthesis were examined by instructing the patient to perform movements in various directions. Necessary adjustments were made and final finishing and polishing was carried out. The patient was instructed on how to remove and place the prosthesis and was advised to remove the prosthesis during night. Cleaning of prosthesis with soap solution was recommended. Recall was done in 1,3 days, 1 week and 1 month. The fit of the prosthesis was to be evaluated every 6 months. ${ }^{4}$

\section{DISCUSSION}

Loss of eye or any other facial structures affect the physical, emotional and psychological well-being of a person. A customized ocular prosthesis can be a viable option for such patients. Accurate impression of the contours of the defect site is required for fabrication of accurately fitting custom made prosthesis. A well made customized ocular prosthesis maintains its orientation when patient performs various movements along
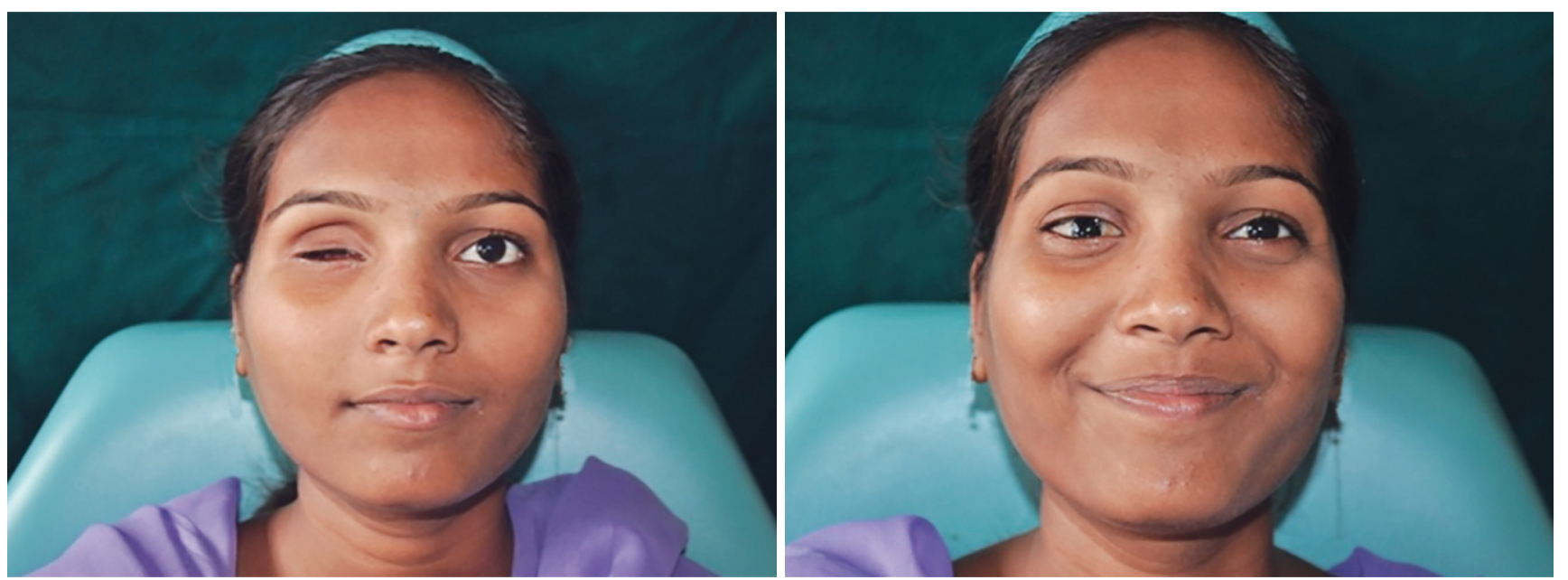

Fig. 17: Preoperative and postoperative view 
with exact fit and esthetics. This instills a sense of self confidence in the patient. Characterization of the ocular prosthesis helps to address the esthetic concern of the patient.

\section{CONCLUSION}

The use of customized prosthesis has been an advantage to the patients who cannot afford implant prosthesis. Also, in the above described case report, the esthetic concern of the patient was addressed by characterization of the prosthesis which was better compared to stock ocular prosthesis. This gave a sense of self confidence to the patient (Fig. 17).

\section{REFERENCES}

1. Mishra SK, Choudhary R. Reproduction of custom made eye prosthesis maneuver: a case report. J Dentist Oral Hygiene 2009 Dec;1(5):59-63.

2. Peyman GA, Sanders DR, Goldbeirg MF. Principles and practice of ophthalmology. New Delhi, Jaypee Brothers Medical Publishers (P) Ltd. 1987;3(2). p. 2334.

3. Somkuwar K, Mathai R, Jose P. Ocular prosthesis: patient rehabilitation: a case report: People's J Scientific Res 2009 July;2(2):21-26.

4. Taylor TD. Clinical maxillofacial prosthetics. Quintessence Publication Co, Inc; 2nd ed. p. 266-276.

5. Poonnanna AA, Tripathi G, Porwal A, Patel M. Art and science behind esthetic ocular prosthesis: a case report. Int J Dent Case Reports 2012;2(5):103-109. 